\title{
Arterial blood gas test to decide whether to reconstruct single or both the arteries in living donor liver transplantation
}

\author{
Seong Hoon Kim, Byung Gon Na, Eung Chang Lee, Sang Jae Park \\ Center for Liver Cancer, National Cancer Center, Goyang-si, Gyeonggi-do, Korea \\ Contributions: (I) Conception and design: SH Kim; (II) Administrative support: SJ Park; (III) Provision of study materials or patients: SH Kim, SJ \\ Park; (IV) Collection and assembly of data: SH Kim, BG Na; (V) Data analysis and interpretation: EC Lee, SH Kim; (VI) Manuscript writing: All \\ authors; (VII) Final approval of manuscript: All authors. \\ Correspondence to: Seong Hoon Kim, MD, PhD. Center for Liver Cancer, National Cancer Center, 323 Ilsan-ro, Ilsandong-gu, Goyang-si, Gyeonggi- \\ do 10408, Korea. Email: kshlj@hanmail.net; kshlj@ncc.re.kr.
}

\begin{abstract}
Background: The graft used in living donor liver transplantation (LDLT) sometimes has two hepatic arteries. This study aimed to introduce arterial-blood gas (ABG) test on the decision on whether to reconstruct a single or both arteries in LDLT.

Methods: Patients with a right lobe graft with two hepatic arteries were enrolled from the prospectively maintained database of our institution. After anastomosis of one of two arteries, the back-bleeding from the second hepatic artery was taken for ABG test. Depending on the results, the second artery was anastomosed or ligated.

Results: Between January 2012 and December 2017, a total of 372 patients underwent LDLT, and 21 living donors had two right hepatic arteries. Based on ABG test results, a single anastomosis was created in 15 recipients while double anastomoses were performed in the remaining 6 recipients. All the patients had an uneventful recovery and were discharged in good conditions. Neither hepatic artery thrombosis nor liver dysfunction was observed during the median follow-up of 26 months (range, 6-71 months). The overall incidence of biliary complications was $9.5 \%(2 / 21)$. Bile leakage arose in one patient with two hepatic artery reconstructions, and the patient subsequently developed biliary stricture. Biliary stricture occurred in another patient with one hepatic artery reconstruction. The biliary complications were successfully managed with endoscopic or percutaneous intervention.
\end{abstract}

Conclusions: ABG test provides a good measure for deciding whether to reconstruct single or both arteries in LDLT.

Keywords: Living donor liver transplantation (LDLT); arterial blood gas test

Submitted Aug 20, 2018. Accepted for publication Oct 10, 2018.

doi: $10.21037 / \mathrm{hbsn} .2018 .10 .04$

View this article at: http://dx.doi.org/10.21037/hbsn.2018.10.04

\section{Introduction}

Hepatic artery reconstruction is one of the critical steps in living donor liver transplantation (LDLT).

The graft used in LDLT sometimes has two hepatic arteries, which can pose a dilemma on whether to reconstruct a single or both arteries. If communication between the two exist, anastomosis of either one is sufficient. Usually the arterial branch of larger diameter is reconstructed first. There have been two criteria for reconstructing the other hepatic artery. If back-bleeding from the other artery is poor, or the arterial flow signals are not detected in all the liver segments of the graft on intraoperative Doppler ultrasonography (DUS), the second artery should be reconstructed. However, the back-bleeding from the cut stump has no objective measurement on how good the backflow is, and so the decision to reconstruct it depends on the surgeon's experience and judgment. 
Furthermore, the back-bleeding can be masked by transient increased arterial resistance that may be attributed to various causes, such as hepatic arterial spasm, increased portal flow, tissue edema, increased cold ischemia time, and an older age in liver donors. And these factors can hinder the clear visualization of hepatic arteries by intraoperative DUS in all the segments of liver graft.

An arterial-blood gas (ABG) test measures the amounts of arterial gases, such as oxygen and carbon dioxide, which we believe is the most obvious method to confirm that the back-bleeding is arterial flow or not. We herein report our experience in introducing $\mathrm{ABG}$ test to decide whether to reconstruct the second hepatic artery in LDLT using the right lobe with two hepatic arteries.

\section{Methods}

We analyzed the database of all donors and recipients undergoing LDLT using a right lobe graft with double arteries at National Cancer Center, Korea from 2012 to 2017. This study protocol was reviewed and approved by the Institutional Review Board of National Cancer Center, Korea (IRB number: NCC2018-0115).

A single in-house surgeon (SH Kim) was a main operator for all the LDLT procedures including donor surgery and hepatic artery anastomosis. The technical details of donor surgery were specified previously $(1,2)$. In the recipient, following total hepatectomy, implantation started with the right hepatic vein anastomosis. Any sizable $(5 \mathrm{~mm}$ or larger in diameter) venous branch of the middle hepatic vein or inferior right hepatic vein of the graft was reconstructed. The right portal vein of the graft was anastomosed to either the right or main portal vein of the recipient. After reperfusion, hepatic artery anastomosis was performed with the use of an interrupted 8-0 nylon suture under a surgical microscope in 1 of 2 arteries. The back-bleeding blood from the cut end of the second hepatic artery was taken into a plastic syringe used for blood gas samples. The sealed syringe was taken to a blood gas analyzer. The sample was transported and kept at room temperature and analyzed within $10 \mathrm{~min}$. If the result of $\mathrm{ABG}$ test was within reference range $\left(\mathrm{pH}\right.$ 7.34-7.44; $\mathrm{PaO}_{2}>90 \mathrm{mmHg} ; \mathrm{PaCO}_{2}$ $35-45 \mathrm{mmHg} ; \mathrm{HCO}_{3}{ }^{-} 22-26 \mathrm{mEq} / \mathrm{L}$ ), the other artery was ligated. Otherwise, the second hepatic artery was reconstructed. Then, bile duct was reconstructed with end-to-end duct-to-duct anastomosis. DUS was performed daily for 10 postoperative days, and liver dynamic computed tomography was checked every 3 months for one year after LDLT.

\section{Results}

During the study period, there were 21 living donors who had two right hepatic arteries. The median donor age was 34 years (range, 20-57 years). There were 13 male and 8 female donors. All the donors recovered uneventfully and none of them required reoperation. The donor median hospital stay was 7 days (range, 7-8 days). No donor had any complications with the median follow-up of 25 months (range, 6-70 months)

The median recipient age was 54 years (range, 43-57 years). There were 15 male and 6 female recipients. The indications for LDLT were hepatocellular carcinoma $(n=13)$, hepatitis B-related cirrhosis $(n=4)$, and fulminant hepatic failure $(n=4)$. The median recipients MELD score was 17 (range, 8-41).

According to ABG test results, a single anastomosis was created in 15 recipients while double anastomoses were performed in the remaining 6 recipients. The median time for each arterial anastomosis was recorded as 15 minutes (range, 8-30 minutes).

All the patients had an uneventful recovery and were discharged in good conditions. Biochemical markers of hepatocyte injury (aspartate aminotransferase and alanine aminotransferase) returned to normal levels by 4 weeks post LDLT. Liver function (bilirubin and international normalized ratio) had recovered by 1 month after LDLT.

DUS and computed tomography for evaluating the liver parenchyma during the follow-up period showed good artery flow without any focal areas of infarction and/or abscess in all patients. Neither hepatic artery thrombosis nor liver dysfunction related to the arterial blood supply was observed during the median follow-up of 26 months (range, 6-71 months).

The overall incidence of biliary complications was $9.5 \%$ $(2 / 21)$. Bile leakage arose in one patient with two hepatic artery reconstructions in the first month post-LDLT, and the patient subsequently developed biliary stricture in postoperative 3 -month. Biliary stricture occurred in another patient with one hepatic artery reconstruction 9 months after LDLT. All the biliary complications were diagnosed as anastomotic ones by cholangiography, which were successfully managed with endoscopic or percutaneous intervention.

\section{Discussion}

The present study was performed to determine whether ABG test could be viewed as a reliable method for deciding 
whether to reconstruct single or both arteries in LDLT. Ideally, with recent advancements in arterial anastomosis techniques, all hepatic arteries could be reconstructed. However, multiple arteries of partial liver grafts usually are of smaller caliber, which can lead to difficult and long anastomoses. Small caliber artery, long anastomosis time, and long total operation time were reported to be risk factors of hepatic artery thrombosis (3). So, single anastomosis would be greatly preferable to multiple anastomoses on the condition that arterial blood supply should be comparable.

The liver grafts are known to have fine, intra-hepatic arterial networks (4). Therefore, an issue has arisen regarding reconstruction of only one of the two hepatic arteries that may maintain minimum arterial flow for the graft to survive. However, there have been no objective criteria on this problem, with conflicting results in the incidence of biliary strictures between single and double anastomoses when the graft has two hepatic arteries $(5,6)$. Actually, in this study, there were no specific characteristics of the graft with regard to double anastomoses.

The overall incidence of biliary stricture reported in this study is lower than those reported in other studies, varying from $13 \%$ to $21 \%(7,8)$, although the small number of patients and the relatively short median follow-up period may adversely affect the validity of statistical comparison.

This is the first report to use ABG test for deciding whether to perform single or double arterial anastomoses in the presence of two hepatic arteries. Unlike the conventional method based on the pulsatile backflow and DUS for the other nondominant artery, ABG test gives objective data on the blood-gas tension values of the arterial partial pressure of oxygen, and the arterial partial pressure of carbon dioxide, and the blood's $\mathrm{pH}$, and shows obvious advantages such as simplicity, effectiveness, and high accuracy. So, we strongly believe that ABG test may be the most obvious way to confirm the presence of intrahepatic arterial communications. This method will be able to find its application not only in left lobe LDLT but also in deceased donor LT using the graft with multiple arteries.

\section{Conclusions}

ABG test provides a simple objective measure for deciding whether to reconstruct a single or both arteries in LDLT.

\section{Acknowledgements}

None.

\section{Footnote}

Conflicts of Interest: The authors have no conflicts of interest to declare.

Ethical Statement: This study protocol was reviewed and approved by the Institutional Review Board of National Cancer Center, Korea (IRB number: NCC2018-0115).

\section{References}

1. Kim SH, Kim YK, Lee SD, et al. The Impact of a Surgical Protocol for Enhanced Recovery on Living Donor Right Hepatectomy: A Single-Center Cohort Study. Medicine (Baltimore) 2016;95:e3227.

2. Kim SH, Kim YK. Living donor right hepatectomy using the hanging maneuver by Glisson's approach under the upper midline incision. World J Surg 2012;36:401-6.

3. Yang Y, Zhao JC, Yan LN, et al. Risk factors associated with early and late HAT after adult liver transplantation. World J Gastroenterol 2014;20:10545-52.

4. Kasahara M, Egawa H, Takada Y, et al. Biliary reconstruction in right lobe living-donor liver transplantation: Comparison of different techniques in 321 recipients. Ann Surg 2006;243:559-66.

5. Uchiyama H, Harada N, Sanefuji K, et al. Dual hepatic artery reconstruction in living donor liver transplantation using a left hepatic graft with 2 hepatic arterial stumps. Surgery 2010;147:878-86.

6. Sugawara Y, Tamura S, Kaneko J, et al. Single artery reconstruction in left liver transplantation. Surgery 2011;149:841-5.

7. Freise CE, Gillespie BW, Koffron AJ, et al. Recipient morbidity after living and deceased donor liver transplantation: findings from the A2ALL Retrospective Cohort Study. Am J Transplant 2008;8:2569-79.

8. Wadhawan M, Kumar A, Gupta S, et al. Post-transplant biliary complications: an analysis from a predominantly living donor liver transplant center. J Gastroenterol Hepatol 2013;28:1056-60.

Cite this article as: Kim SK, Na BG, Lee EC, Park SJ. Arterial blood gas test to decide whether to reconstruct single or both the arteries in living donor liver transplantation. HepatoBiliary Surg Nutr 2018;7(6):440-442. doi: 10.21037/hbsn.2018.10.04 